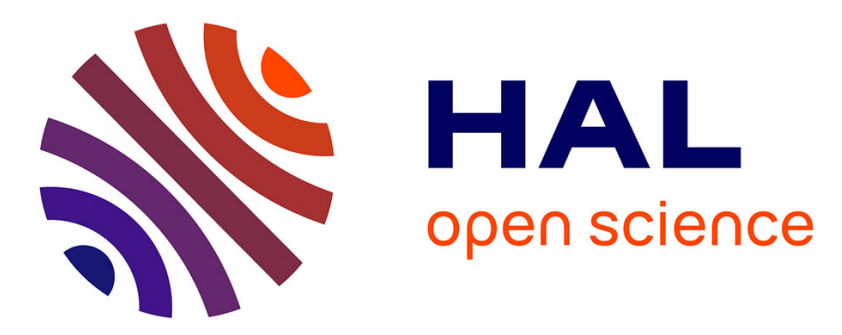

\title{
Sur-éducation et déclassement socioprofessionnel dans l'accès à l'emploi : état des lieux des publications nord-américaines et françaises
}

Vincent Chabault

\section{- To cite this version:}

Vincent Chabault. Sur-éducation et déclassement socioprofessionnel dans l'accès à l'emploi : état des lieux des publications nord-américaines et françaises. Revue des sciences de l'education, 2008, 34 (1), pp.185-202. hal-00637118

\section{HAL Id: hal-00637118 \\ https://hal.science/hal-00637118}

Submitted on 3 Nov 2011

HAL is a multi-disciplinary open access archive for the deposit and dissemination of scientific research documents, whether they are published or not. The documents may come from teaching and research institutions in France or abroad, or from public or private research centers.
L'archive ouverte pluridisciplinaire HAL, est destinée au dépôt et à la diffusion de documents scientifiques de niveau recherche, publiés ou non, émanant des établissements d'enseignement et de recherche français ou étrangers, des laboratoires publics ou privés. 


\title{
Article
}

\section{"Sur-éducation et déclassement socioprofessionnel dans l'accès à l'emploi : état des lieux des publications nord-américaines et françaises »}

\author{
Vincent Chabault \\ Revue des sciences de l'éducation, vol. 34, n 1, 2008, p. 185-202.
}

Pour citer la version numérique de cet article, utiliser l'adresse suivante :

http://id.erudit.org/iderudit/018996ar

Note : les règles d'écriture des références bibliographiques peuvent varier selon les différents domaines du savoir.

Ce document est protégé par la loi sur le droit d'auteur. L'utilisation des services d'Érudit (y compris la reproduction) est assujettie à sa politique d'utilisation que vous pouvez consulter à l'URI http://www.erudit.org/documentation/eruditPolitiqueUtilisation.pdf

Érudit est un consortium interuniversitaire sans but lucratif composé de l'Université de Montréal, l'Université Laval et l'Université du Québec à Montréal. Il a pour mission la promotion et la valorisation de la recherche. Érudit offre des services d'édition numérique de documents scientifiques depuis 1998.

Pour communiquer avec les responsables d'Érudit : erudit@umontreal.ca 


\title{
Sur-éducation et déclassement socioprofessionnel dans l'accès à l'emploi : état des lieux des publications nord-américaines et françaises
}

\author{
Vincent Chabault, doctorant \\ École des hautes études en sciences sociales, Paris
}

\begin{abstract}
RÉSUMÉ - Depuis au moins deux décennies, le phénomène de déclassement (ou sur-éducation) dans l'accès à l'emploi caractérise l'insertion professionnelle des jeunes populations, en France comme en Amérique du Nord. Dans cette perspective, nous dressons un état de lieux des publications nord-américaines et françaises à ce sujet. Après avoir présenté les différentes approches visant à mesurer ce phénomène, nous exposons les résultats d'enquêtes statistiques canadiennes, américaines et françaises, depuis les années 1970 jusqu'aux années 2000.
\end{abstract}

MOTS CLÉS • insertion professionnelle, jeunes générations, diplômes, déclassement, France, Amérique du Nord.

Le changement ayant été rapide, on peut voir les mêmes individus ou les mêmes groupes qui affirmaient, il y a peu, que la "démocratisation de la culture» serait réalisée «lorsque le jardinier pourrait lire Platon dans le texte", se voiler aujourd'hui la face en constatant qu'on risque de se retrouver jardinier avec une licence de grec... (Passeron, 1982, p. 551).

\section{Introduction}

Depuis les deux dernières décennies, en Amérique du Nord comme en France, l'insertion professionnelle des jeunes populations se caractérise par la croissance des phénomènes de déclassement (ou sur-éducation ${ }^{1}$ ) dans l'accès à l'emploi. Dans cette perspective, nous proposons un état des lieux des publications françaises et nordaméricaines, traitant de l'écart qui existe entre le niveau scolaire atteint par un individu lors de sa formation initiale et les qualifications requises par l'emploi qui occupe $^{2}$. Le choix de l'Amérique du Nord et de la France se justifie par la volonté de mettre au jour des similitudes en ce qui concerne les dynamiques d'insertion professionnelle des jeunes générations. Il ne s'agit pas ici d'expliquer les raisons de la croissance du niveau d'études de telle ou telle population, mais d'exposer les analyses de ce phénomène effectuées par des économistes et des sociologues français et nordaméricains depuis les années 1970. En Amérique du Nord et en France (mais aussi dans d'autres pays européens sur lesquels nous reviendrons plus brièvement), la 
sur-éducation n'est pas un fait étudié récemment. Elle est traitée depuis le développement massif de la scolarisation adolescente et estudiantine dans les sociétés industrialisées de la seconde moitié du $\mathrm{xx}^{\mathrm{e}}$ siècle.

Tout d'abord, nous présentons les différentes approches de mesure du déclassement; nous distinguons ensuite, de manière chronologique, les publications des années 1970 de celles des années 1980, 1990 et 2000. Cette première distinction ne renvoie pas aux mêmes générations investiguées: celles issues du baby-boom accédant à l'emploi avant 1980, et celles postérieures ayant connu la seconde explosion scolaire (Chauvel, 1998a). Nous différencions également les publications américaines rédigées davantage par des économistes du travail et de l'éducation, des articles français et canadiens qui relèvent de sociologues et de statisticiens. Si l'on excepte les travaux de Burris, une partie des travaux canadiens et français se distinguent par le fait qu'ils cherchent à interpréter le phénomène du déclassement, à saisir ses effets dans l'emploi (Vultur, 2006), dans le travail (Beaud, 2000), dans l'action collective (Burris, 1983; Saint-Pierre, 2001), et à observer les conséquences de l'allongement des parcours scolaires sur la stratification de la société (Chauvel, 1998b; Langlois, 2003; Schwartz, 1998). De leur côté, les travaux américains se concentrent davantage sur les résultats au niveau des salaires et de la productivité des travailleurs (Berg, 1970; Sicherman, 1991). Parallèlement à la présentation synthétique de ces travaux, nous exposons les résultats des investigations les plus récentes.

Même si nous avons privilégié les travaux statistiques sur de larges populations, notons que quelques travaux qualitatifs, comme ceux de Beaud (2000) sur les jeunes générations ouvrières ou ceux de Cartier (2003) sur les facteurs, apportent un éclairage inédit et intéressant aux effets du déclassement sur le rapport au travail des dominés aux études longues (Schwartz, 1998).

\section{Mesurer le déclassement : intérêt des enquêtes et diversité des approches}

Depuis le début des années 1980 en France comme en Amérique du Nord, le marché du travail des jeunes générations (18-30 ans) a connu des transformations fondamentales. Du côté de l'offre de travail, le taux d'activité diminue et le niveau général de formation augmente; du côté de la demande, les exigences en termes de qualifications et de compétences s'élèvent et les débutants sont moins embauchés qu'auparavant. En parallèle, le déclassement se développe. Être déclassé signifie posséder un niveau de formation a priori supérieur à celui requis pour l'emploi occupé. Les enquêtes sur le lien entre la formation et l'emploi offrent un triple intérêt (Forgeot et Gautié, 1996, 1997). Au niveau macroéconomique, elles permettent d'étudier le chômage des jeunes, et, au-delà, celui des personnes non qualifiées. Ces enquêtes contribuent également à expliquer les différentiels de salaire, notamment selon l'âge et le niveau d'études. Enfin, à un niveau microéconomique, l'analyse du phénomène permet de mieux cerner, à la fois, les comportements de demande de travail des employeurs et d'offre de la main-d'œuvre juvénile. 
La mesure et l'analyse du déclassement supposent la mise en relation d'une nomenclature des niveaux de formation et d'une nomenclature des niveaux d'emploi. Trois approches ont été utilisées jusqu'à présent: les approches adéquationniste, statistique et subjective.

Dans une perspective dite adéquationniste, on compare emplois, compétences requises et formations acquises selon le niveau de spécialité. On établit alors, $a$ priori, une table de correspondances entre les professions censées exiger certaines compétences et les diplômes susceptibles de résumer les compétences acquises, et on la compare à la situation observée. Il reste ensuite à déterminer les limites à partir desquelles on peut parler de déclassement. On dit alors qu'une personne est déclassée lorsque la formation acquise est d'un niveau supérieur à celui exigé par l'emploi. Utilisée dans les années 1970 (Affichard, 1981), cette méthode suppose l'existence d'un lien précis entre formation et emploi. Or, cette concordance n'est ni automatique ni spontanée ${ }^{3}$. Pour cette raison, d'autres auteurs ont recours à la méthode dite statistique. Elle consiste à définir une norme a posteriori, basée sur la nomenclature des catégories professionnelles, en fonction des correspondances effectivement observables à un moment donné, puis à évaluer les évolutions sur une période donnée. Il existe là aussi des soucis liés à la méthode. Du côté des emplois, des effets de nomenclature peuvent être observés, c'est-à-dire qu'un même emploi conservant le même classement peut exiger de nouvelles compétences du fait du progrès technique, des changements de formation (un cursus peut être sanctionné par un même diplôme et voir son contenu se dégrader), et des effets de structure inhérents aux transformations des activités (Rose, 1998, p. 87). Enfin, l'approche dite subjective repose sur le sentiment des individus à l'égard de leur emploi. C'est le travailleur qui évalue le niveau de qualifications nécessaire pour son emploi et qui détermine également si son emploi correspond ou non à son propre niveau d'éducation. Cette méthode comporte également des lacunes, puisqu'elle repose uniquement sur le témoignage des enquêtés qui ne sont pas confrontés à des données structurelles (analyse des postes de travail, conventions collectives, etc.).

La méthode adéquationniste a surtout été utilisée aux États-Unis et en France au début des années 1980. La méthode statistique n’a été mobilisée qu’en France, tandis que la méthode subjective a été employée à de nombreuses reprises en Amérique du Nord, tout en restant très marginale en France (Lefresne, 2003, p. 43). Selon les méthodes et pour les mêmes années et les mêmes populations investiguées, des différences apparaissent. L'usage de la méthode statistique et de la méthode subjective lors de l'enquête Génération 98 du Céreq 4 indique, par exemple, que les jeunes femmes se déclarent moins souvent déclassées que les jeunes hommes, alors même qu'elles le sont plus souvent, d'après l'approche statistique.

Abordons, dans un premier temps, les travaux pionniers qui concernent ce phénomène. 


\section{Le phénomène de sur-éducation entre l'après-guerre et les années 1980: les premières investigations}

Les premières enquêtes sur le déclassement sont l'œuvre d'économistes américains et datent des années 1970. Cette période est marquée par l'accès des enfants du baby-boom au marché de l'emploi, dans un contexte où l'adaptation aux nouvelles technologies nécessite un personnel hautement qualifié (Smith, 1986, p. 90).

Les travaux visent à mesurer les conséquences du recrutement de personnes surqualifiées sur la productivité et le montant des salaires. Pour Berg (1970), en raison d'un manque de motivation et d'intérêt, les travailleurs surqualifiés sont moins productifs que ceux pour qui l'emploi correspond à la formation initiale. Les travailleurs surqualifiés reçoivent des salaires plus faibles que les autres à cause de ce manque de motivation. Par ailleurs, l'auteur observe que la croissance de l'offre de travailleurs ayant un niveau de scolarité collégial durant les années 1970 n’a pas été suivie par une augmentation de la demande. D’après lui, les employeurs en ont profité pour élever le minimum de qualifications fixé pour des emplois qui n'auraient théoriquement pas requis un tel niveau de savoirs et de savoir-faire. Cette analyse est reprise quelques années après par Freeman (1976): l'offre de travailleurs hautement qualifiés a augmenté pendant un moment où la demande pour ces travailleurs était en baisse, forçant une majorité d'entre eux à accepter des emplois pour lesquels le niveau de qualifications requis était plus faible.

De son côté, Thurow envisage l'embauche de personnes surqualifiées comme un mode de gestion rationnel du personnel. Il considère que l'employeur utilise la qualification de son personnel comme signal du montant de formation professionnelle qu'il devra ensuite investir pour la personne qu'il recrute. Bref, si l'employeur embauche des personnes surqualifiées, c'est pour minimiser, dans le futur, les dépenses de formation pour son personnel (Thurow, 1975). L'entreprise emploie alors ceux dont la formation est moindre, et les plus aptes à être formés le plus rapidement. Thurow développe également le modèle de job competition (concurrence pour l'emploi). Il s'appuie sur deux hypothèses: l'équilibre entre l'offre et la demande de travail se réalise par les quantités d'emplois, et la productivité est une caractéristique de l'emploi et non du travailleur. Pour un emploi, les demandeurs forment une file d'attente (labor queue) au sein de laquelle les employeurs puisent en fonction de leurs besoins et selon les critères de diplôme et d'expérience. L'employeur va classer les candidats à l'emploi par coûts de formation croissants dans une file d'attente: les plus diplômés, pour qui les coûts d'acquisition de la formation sont les plus bas, se situent en tête de file.

Au tournant des années 1970 et 1980, d'autres travaux ont été réalisés sur les conséquences du déclassement au niveau des attitudes au travail et des comportements politiques. En France, Bourdieu développe la thèse de la déqualification structurale. Cette déqualification concerne les membres d'une génération voués à obtenir de leurs titres scolaires moins que n'en aurait obtenu la génération précédente. Est déclassée, toute personne contrainte de renoncer au classement social 
qu'elle pensait obtenir grâce à son diplôme. Ce phénomène entraîne une sorte de désillusion collective et une attitude de désaffection à l'égard du travail (Bourdieu, 1978, p. 9). Aux États-Unis, l'enquête de Burris (1983) confirme que l'overeducation peut conduire à l'insatisfaction au travail et à la contestation politique. Selon lui, toutefois, le sentiment d'amertume s'exprime davantage de façon personnelle que par une action collective.

L'ensemble des études que nous allons aborder maintenant concerne davantage les générations d'individus entrant sur le marché de l'emploi à partir des années 1980. En Amérique du Nord comme en France, les investigations deviennent plus nombreuses au fur et à mesure que le phénomène de déclassement augmente, et qu'il devient un débat de société, au-delà du seul champ de la recherche universitaire ${ }^{5}$.

\section{Les enquêtes sur la sur-éducation depuis les années 1980}

\section{Les travaux nord-américains}

En mobilisant l'approche subjective, Sicherman (1991) observe que $41 \%$ des travailleurs de son échantillon se disent surqualifiés, et $16 \%$ sous-qualifiés. En moyenne, les travailleurs surqualifiés gagnent $4 \%$ de plus que ceux adéquatement qualifiés, alors que les sous-qualifiés gagnent 1,70\% de moins. Le personnel surqualifié perçoit un salaire plus élevé que ses collègues, mais il gagne moins que celui doté d'un niveau de qualification similaire, qui travaille dans un emploi dont le niveau de qualifications équivaut à celui qui est requis. Quant aux travailleurs sous-qualifiés, ils reçoivent des salaires plus faibles que leurs collègues, mais plus élevés que les travailleurs avec le même niveau de qualifications, qui ont des emplois requérant leur niveau de formation. Les travailleurs surqualifiés sont souvent plus jeunes, ont moins d'expérience mais, comme ils ont atteint un niveau de formation initiale élevé, le budget nécessaire à leur formation professionnelle reste faible. Enfin, cette catégorie de personnel a un plus haut taux de mobilité et offre plus de capacité à aller vers des occupations de plus haut niveau et à connaître ainsi le reclassement. Avec l'ancienneté, les situations de déclassement sont donc en diminution (Sicherman, 1991).

L'article de Guérin, Carrière et Wils (1999) est l'un des rares à traiter du phénomène de démobilisation dans le travail chez les jeunes diplômés universitaires. Un questionnaire d'enquête a été diffusé auprès de 2500 jeunes professionnels diplômés au cours de l'année 1993 de l'École Polytechnique, de l'École des Hautes Études Commerciales (HEC) et dans les départements proposant des formations professionnalisantes de l'Université de Montréal. L'accent y est mis sur les attentes professionnelles et les conditions de possibilité de ces attentes. Ce n'est pas tant la différence entre les aspirations élevées observées à l'embauche et la réalité quotidienne du travail, c'est-à-dire la non-réalisation des attentes initiales, qui génère la démobilisation des jeunes. C'est plutôt le manque d'opportunités offertes par 
l'organisation qui permet d'expliquer la faible productivité et la démotivation dans le travail chez ces jeunes recrues; ces dernières détestent par ailleurs que leurs compétences soient sous-utilisées (Guérin, Carrière et Wils, 1999, p. 647 et 666).

À partir de données issues d'enquêtes menées à deux reprises sur trois cohortes de diplômés canadiens, l'étude de Frenette (2000) tente de répondre à la question suivante: Dans quelle mesure le niveau de scolarité des diplômés a-t-il dépassé le besoin des employeurs? Les investigations citées mobilisent une approche spécifique, puisque les personnes interrogées étaient soumises à l'interrogation suivante: Lorsqu'on vous a choisi pour cet emploi, quelles étaient les exigences scolaires minimales pour répondre aux critères d'embauche? Ce sont les exigences de l'employeur qui ont été ici recueillies, puis comparées au niveau scolaire des travailleurs. Près de $27 \%$ à $48 \%$ des titulaires d'un diplôme d'études collégiales, d'un baccalauréat ou d'un doctorat sont surqualifiés au Canada. La situation de ces diplômés diminue dans les années qui suivent l'accès à l'emploi, et ce pour tous les niveaux, à l'exception des études collégiales (Frenette, 2000).

La surqualification varie selon le sexe: parmi les diplômés d'études collégiales, les hommes sont davantage soumis à la surqualification que les femmes. Dans le cycle universitaire, cependant, les hommes sont légèrement moins touchés par la surqualification au niveau du baccalauréat: $23 \%$ chez les hommes contre $28 \%$ chez les femmes pour la cohorte de 1990 interrogée en 1995. Pour ce qui est de la maîtrise, les parts de surqualifiés sont plus importantes chez les hommes que chez les femmes. Au niveau du doctorat, ce sont les femmes qui connaissent un taux de surqualification plus élevé (30\% en 1995 pour la cohorte 1990, contre $26 \%$ chez les docteurs masculins).

Enfin, les taux de surqualification varient selon les spécialités de formation. Au niveau collégial, les diplômés en soins infirmiers et en technologies médicales ne sont que très peu en situation de déclassement. C'est du côté des diplômés en art et sciences humaines, autres sciences de la santé, sciences naturelles et zootechnie, services de protection, services de secrétariat et autres services en entreprises que les taux de surqualifiés sont les plus hauts: la part varie entre 30 et $62 \%$ selon les champs d'études. Pour le niveau baccalauréat, des différences selon le sexe sont constatées: les hommes formés en mathématiques et sciences physiques sont davantage surdiplômés que les femmes en 1995 pour la cohorte 1990 (23\% contre 9\%). La situation est équivalente pour les autres sciences sociales (38\% pour les hommes, $27 \%$ pour les femmes) et pour les sciences agricoles et biologiques ( $30 \%$ et $23 \%$ ). Pour toutes les cohortes, ce sont les bacheliers et les bachelières formés en beaux-arts et en sciences humaines qui sont les plus surqualifiés dans l'emploi. Viennent ensuite les individus des disciplines suivantes: les autres sciences sociales pour les deux sexes, l'économie pour les hommes et les sciences agricoles et biologiques pour les femmes en 1995 (Frenette, 2000, p. 15). Pour ce qui est de la maîtrise, les diplômés ont tendance à afficher des taux importants de surqualification dans les disciplines telles que les beaux-arts, les sciences humaines et sociales, 
mais également l'informatique et le commerce, pour les femmes. C'est l'inverse qui se produit pour les titulaires de maîtrise en sciences de la santé. En ce qui concerne le doctorat, les taux de surqualification se divisent par trois ou quatre par rapport au taux du baccalauréat: entre 3 et $18 \%$. Les docteurs en mathématiques sont davantage surqualifiés en 1995. Puis viennent les hommes et les femmes titulaires de doctorat en éducation, les plus touchés, avec des taux de 13 et $14 \%$. Les docteurs en sciences humaines et en beaux-arts se placent également sur le podium, avec un taux de $12 \%$ chez les hommes et $10 \%$ chez les femmes (Frenette, 2000, p. 17).

Les pourcentages de surdiplômés sont sensiblement équivalents dans le privé et dans le public. L'ensemble des résultats laisse entendre que les diplômés des cycles supérieurs sont moins susceptibles d'être surqualifiés, sauf pour les titulaires d'une maîtrise. Cependant, compte tenu de l'inflation des taux de recrutement, certains occupent bien souvent un emploi pour lequel le baccalauréat seulement est requis. Ce sont alors, pour ces mêmes emplois, les bacheliers qui sont victimes de l'effet d'éviction ou de report dont parlent Forgeot et Gautié (1996, p. 8).

Par ailleurs, les données montrent que les personnes ayant un niveau de scolarité plus élevé que l'emploi ne le requiert ont généralement une rémunération supérieure à celle des autres travailleurs (Frenette, 2000, p. 11). Enfin, dans le cas des études collégiales et du baccalauréat, les diplômés surqualifiés utilisent moins leurs compétences que les diplômés non surqualifiés. Chez les titulaires d'un diplôme de deuxième ou de troisième cycle, la situation de surqualification n'entraîne pas une diminution importante de l'utilisation des compétences. En d'autres termes, les résultats complémentaires de l'enquête indiquent que les savoirs et savoir-faire scolaires sont mobilisés par les surdiplômés, quel que soit le type d'emploi occupé.

Reprenant le titre de l'ouvrage de Berg (1970), l'économiste Vahey (2000) traite de la disparité entre le niveau d'éducation et les qualifications requises pour un emploi, en mobilisant les données issues de l'enquête National Survey of Class Structure and Labour Process in Canada de 1982. La mesure du déclassement est ici subjective: les personnes interrogées sont soumises à des questions du type Quel est le plus haut niveau de formation que vous avez terminé? ou Quel type de formation scolaire est normalement requis pour des personnes qui font le même type de travail que vous? (Vahey, 2000, p. 220) [notre traduction]. Tout d'abord, $30 \%$ des hommes se disent surqualifiés, alors que $24 \%$ se disent sous-qualifiés, et que $32 \%$ des femmes considèrent qu'elles sont surqualifiées et $17 \%$ sous-qualifiées. Les résultats montrent que le phénomène de sur-éducation dans l'emploi touche en premier lieu les jeunes travailleurs: $57 \%$ des hommes et 33\% des femmes de moins de 26 ans sont surqualifiés. Ensuite, selon l'auteur, cette situation de sur-éducation, pour les hommes, a un rendement positif dans l'emploi, même si ces rendements dépendent des niveaux de qualifications requis pour l'emploi occupé. Les résultats pour les femmes different de ceux des hommes: les rendements sont non significatifs pour tous les niveaux de 
qualifications requis. En d'autres termes, l'analyse de Berg est ici rejetée pour les femmes comme pour les hommes: les travailleurs surqualifiés ne sont pas moins productifs et leurs salaires ne sont pas moins élevés que les travailleurs dont les qualifications sont en adéquation avec leur emploi.

Dans leur enquête sur les travailleurs du Québec et de l'Ontario en 1991 et 1996, Montmarquette et Thomas (2003) observent que le nombre de personnes adéquatement qualifiées connaît une augmentation significative. Autrement dit, il existe un meilleur ajustement entre le diplôme des individus et le niveau d'études requis pour l'emploi en 1996, tous secteurs d'activité confondus.

C'est en atteignant un niveau d'études universitaire que la surqualification diminue; cela signifie que les individus sont plus touchés par le déclassement s'ils ont mis un terme à leurs études au niveau secondaire et collégial. Entre les deux provinces, le Québec se distingue: la percée de la qualification adéquate y est moins importante qu'en Ontario, et le pourcentage de personnes surqualifiées y est plus important (22\% en 1996). Si, pour les individus ayant atteint le niveau universitaire, le nombre de personnes surqualifiées a chuté et est équivalent dans ces deux provinces canadiennes, on compte nettement plus de personnes sur-éduquées au Québec qu'en Ontario pour le niveau collégial, en 1996. Pour le secondaire, le nombre de surqualifiés est plus important au Québec, mais diminue entre 1991 et 1996, où il passe de $52 \%$ à $39 \%$ (Montmarquette et Thomas, 2003, p. 12-14).

En s'intéressant aux caractéristiques des individus, les auteurs montrent que les personnes sous-qualifiées sont les plus âgées (moyenne 43 ans). Inversement, les personnes surqualifiées sont les plus jeunes (moyenne 34 ans): le manque d'expérience est compensé par les diplômes. Leur enquête montre enfin que la surqualification est plus fréquente chez les hommes que chez les femmes, alors que ces mêmes auteurs avançaient l'hypothèse que les femmes seraient surqualifiées en raison de la discrimination dont elles sont victimes sur le marché du travail.

Dans un ouvrage collectif traitant de la jeunesse au Québec, Vultur (2003) montre que les jeunes, diplômés ou non, manifestent une attitude assez positive face à l'activité professionnelle et considèrent leur carrière professionnelle comme l'une des composantes essentielles de leur vie. Malgré ces résultats, le taux de chômage des détenteurs d'un diplôme universitaire de la catégorie des 15-24 ans est passé de $7,10 \%$ à $8,40 \%$, et celui des titulaires d'un baccalauréat de $7,30 \%$ à $8,90 \%$. Non seulement la possession de titres universitaires ne garantit pas l'accès à un emploi qualifié mais, pour les diplômés, la stabilisation dans l'emploi qualifié se fait de plus en plus de manière indirecte, après des périodes de chômage, d'intérim ou de contrat à durée déterminée. Si la fragilisation des emplois qualifiés reste moins forte que la précarisation des emplois peu ou non qualifiés, il n'en existe pas moins une érosion de l'invulnérabilité offerte jadis par les diplômes (Vultur, 2003, p. 66). Une scolarité longue ne suffit pas à garantir une insertion dans un emploi stable, en général, et dans un emploi qualifié en adéquation avec la formation initiale suivie, en particulier. 
Dans un article plus récent, Vultur (2006) analyse la hausse du nombre de diplômés au Québec et la façon dont les jeunes s'insèrent dans les différentes catégories professionnelles. Il expose également les causes macroéconomiques du déclassement et tente de saisir les attitudes d'adaptation des jeunes face à la dévalorisation des diplômes. Comme en France, les politiques visant à offrir au plus grand nombre l'égalité des chances par l'accès à l'éducation, ainsi que les besoins en main-d'œuvre qualifiée, ont conduit à l'augmentation du nombre de diplômés depuis les trois dernières décennies. Si l'on retient le seul taux d'accès au baccalauréat, celui-ci a connu une hausse de près de 20\% de 1984-1985 à 2002-2003. Le taux d'obtention du baccalauréat est passé de $15 \%$ en 1976 à $29 \%$ en 1996 (Vultur, 2006, p. 43). Depuis le milieu des années 1970, le nombre de diplômés au Québec et le taux de diplomation ont considérablement augmenté: la quantité de personnes peu ou non diplômées a chuté considérablement, et le niveau d'études atteint est de plus en plus élevé. Néanmoins, la croissance du niveau d'études de la population connaît un certain fléchissement depuis le milieu des années 1990, et selon les types de formation. Ce phénomène peut signifier un simple mouvement conjoncturel ou l'atteinte d'un niveau maximum d'éducation sur une population, et s'expliquer par des compressions budgétaires à l'Université. On doit également l'analyser en tenant compte des attitudes des jeunes face aux études et à l'état du marché de l'emploi. En effet, une forte croissance économique dans l'histoire du marché de l'emploi caractérise la fin des années 1990 et le début des années 2000 au Québec: en effet, depuis 1997, il s'est créé plus de 440000 emplois, dont 118000 en 2002.

En ce qui concerne l'afflux des diplômés universitaires au sein des catégories professionnelles, leur part est en croissance depuis la fin des années 1990 dans les secteurs liés à la gestion, aux finances, à l'administration, à la santé, au transport et à la machinerie, à la fabrication et aux services d'utilité publique. C'est donc au sein des secteurs d'activités les plus soumis au développement des nouvelles technologies et au renouvellement organisationnel que le niveau moyen d'études des travailleurs a crû. Inversement, la population des diplômés universitaires diminue au sein des professions liées aux sciences naturelles, aux sciences sociales, à l'administration publique, à la religion et dans la vente et les services. La baisse de la proportion de diplômés universitaires reste toutefois très légère: 5,60 \% de bacheliers en 1996 dans le secteur vente et services contre 4,80\% en 2001 (Vultur, 2006, p. 48).

Face au constat de l'augmentation du nombre des individus (fortement) diplômés au Québec et à leur entrée sur le marché de l'emploi, Vultur (2006) s'interroge sur la relation formation/emploi et sur les mécanismes d'absorption du flux des diplômés. Les besoins du marché du travail québécois justifient-ils cette hausse générale du niveau d'études?

Après avoir évoqué les différentes approches interprétatives de la sur-éducation et présenté les principaux résultats d'enquêtes antérieures sur les surqualifiés 
québécois, l'auteur s'intéresse aux stratégies d'adaptation des jeunes face au phénomène de déclassement. Le déclassement est généré par la croissance du nombre de diplômés du postsecondaire et par l'élévation du niveau d'études moyen atteint par les individus. Ce phénomène est aussi lié au progrès technique et aux nouvelles formes d'organisation du travail, qui se traduisent par la hausse du niveau de compétences requises pour un poste. Les recruteurs recherchent alors un personnel doté de compétences acquises lors de la formation initiale pour des emplois qualifiés. La situation de sur-éducation provient plus précisément du décalage qui existe entre l'élévation des qualifications scolaires des individus et la croissance insuffisante du nombre de postes qualifiés. Par conséquent, les exigences des employeurs deviennent souvent supérieures aux compétences requises pour un poste, et ceux-ci vont puiser dans le début de la file d'attente où sont placés les candidats les plus diplômés.

L'analyse de la sur-éducation doit être envisagée dans un cadre plus large: celui de la dévalorisation des titres scolaires due à l'inflation de la demande scolaire. Le seul diplôme ne garantit pas un niveau de rémunération ou une adéquation formation-emploi à tous les individus. Dans ce contexte, certains jeunes vont choisir d'autres moyens de signaler leurs compétences sur le marché du travail et vont mobiliser d'autres formes de reconnaissance de leur qualifications: poids accordé à l'expérience et mise en valeur des caractéristiques de personnalité (Vultur, 2006, p. 63). Ces attitudes conduisent souvent à l'abandon des études. Par exemple, en 2001-2002, 43 \% des élèves de l'enseignement professionnel au niveau secondaire ont quitté les études sans diplôme et sans s'y réinscrire ultérieurement. Les effets de l'expérience viendraient alors concurrencer les rendements de plus en plus incertains du titre scolaire.

Pour Vultur, il est hors de question de remettre en cause les politiques éducatives conduisant à la croissance importante des diplômés et à leur diffusion au sein des catégories professionnelles. Toutefois, si les bénéfices du système d'éducation sont importants pour un pays et sa population, il est nécessaire de réfléchir sur la nature de la formation et sur la manière de former les jeunes générations, c'est-à-dire qu'il faut s'intéresser de près à la nature des diplômes ${ }^{6}$, à leur niveau et aux besoins de l'économie, en raison notamment de l'augmentation du nombre de surdiplômés et du coût des politiques éducatives. Ces propos rejoignent ceux de Duru-Bellat (2006) pour la France. Il n'y a aucune contradiction à dénoncer, d'un côté, les effets pervers d'une massification scolaire et, d'un autre, à reconnaître que l'élévation du niveau général de formation est une bonne chose à long terme pour une société.

Abordons maintenant les travaux français.

\section{Les travaux français sur le déclassement}

Pour chaque profession et pour chaque niveau de diplôme, on assiste en France à une forte augmentation générale du déclassement depuis une vingtaine d'années, 
et tout spécialement dans la période 1992-1995, chez les jeunes actifs occupés de 18-29 ans. La part des surdiplômés s'est fortement étendue entre 1986 et 1995 pour les titulaires d'un diplôme de niveau bac (fin du secondaire) et au-delà. Elle augmente de près de six points chez les titulaires d'un diplôme de grande école ou de troisième cycle. Multipliée par 2,6 en neuf années chez les diplômés de deuxième cycle, elle s'est accrue de près d'un quart chez les diplômés de l'enseignement supérieur court, et de plus de la moitié chez les titulaires d'un baccalauréat général ou d'un brevet de technicien. Enfin, on la trouve multipliée par près de 2,4 chez les titulaires d'un bac professionnel ou technique. D'après Forgeot et Gautié (1996, p. 9), la baisse de l'emploi des jeunes et la modification de sa structure, accompagnées de l'afflux massif de diplômés, provoquent un phénomène de report en termes de recrutement. Si, pour tel poste, l'usage était de recruter à un niveau bac, les individus scolarisés jusqu'à des paliers supérieurs au bac seront désormais favorisés. Dans ce cas, les peu ou non-diplômés deviennent alors des victimes de la dévaluation des titres scolaires et d'un effet d'éviction à l'embauche. Alors que, pour les non ou peu qualifiés, ces conséquences se traduisent par une situation de chômage, la raréfaction de l'emploi se manifeste pour les plus diplômés par une redistribution des emplois.

Les surdiplômés sont surtout des femmes et des débutants: plus de $24 \%$ des jeunes femmes sont surdiplômées contre moins de $18 \%$ de jeunes hommes. En moyenne, les débutants sont (logiquement) davantage surdiplômés. De fait, quel que soit le niveau de formation, le phénomène de reclassement est lié à l'ancienneté sur le marché du travail.

Gautié et Nauze-Fichet (2000) prolongent les mesures du déclassement en France sur la période 1990-2000. Ils précisent avant toute chose que la France se caractérise par un fonctionnement de type marché interne: les diplômes, acquis à l'extérieur de l'entreprise, servent de critère de recrutement, mais c'est l'expérience acquise à l'intérieur de l'entreprise qui transformera cette compétence "potentielle» en compétence reconnue dans les qualifications (p. 278). En d'autres termes, le système de formation est un filtre permettant de classer les individus selon leurs aptitudes, et leur diplôme est davantage considéré comme un signal ${ }^{7}$ des potentialités des individus que comme une mesure des compétences directement productives. Si le déclassement poursuit sa croissance jusqu'à la seconde moitié des années 1990 chez les 18-29 ans, en particulier pour les diplômés de niveau baccalauréat (la part des déclassés double) et de niveau deuxième cycle universitaire (elle augmente de 80 \%), la tendance commence à s'infléchir depuis 1997-1998, mais uniquement pour les seuls diplômés des Grandes Écoles ou du troisième cycle universitaire. Ce faible changement correspond à une reprise de la croissance (p. 281). Le reclassement survient alors d'autant plus tôt que le niveau d'études est élevé chez les individus surqualifiés. C'est également le tassement de la croissance du nombre de diplômés qui permet d'expliquer la diminution du déclassement pour certaines catégories, comme celle des diplômés des Grandes Écoles et 
du troisième cycle à la fin des années 1990. Pour les autres diplômés du supérieur qui ne sont pas touchés par cette inflexion, c'est en raison du maintien de leur augmentation numérique qu'une baisse du déclassement n'est pas constatée (plus de $20 \%$ pour le deuxième cycle et plus de $10 \%$ pour le premier cycle entre 1998 et 2000). Gautié et Nauze-Fichet (2000) s'intéressent enfin à la part des diplômés des Grandes Écoles et du troisième cycle récemment employés sur des postes d'ouvriers ou employés. Après une progression très forte entre 1991 et 1996, on assiste à une faible diminution de leur présence dès 1997. Ils représentent cependant $10 \%$ des membres de ces professions en 1997, et $9 \%$ en 1999. Les auteurs insistent sur l'importance de s'intéresser à la nature des diplômes, et pas uniquement à leur niveau, lorsque l'on s'interroge sur leur adéquation aux besoins de l'entreprise. De telles enquêtes devraient être complétées par des études menées auprès d'entreprises tout en prenant en compte les réponses des employeurs avec prudence: l'explosion scolaire et l'atonie de l'emploi en France leur permettent de recruter des individus fortement diplômés sans que les caractéristiques du poste de travail et son statut ne le justifient.

Le diplôme joue encore en France un rôle déterminant en début de carrière lors de l'entrée sur le marché du travail, mais c'est pourtant en début de carrière, du moins jusqu'en 1998-1999, que les relations entre le diplôme et l'emploi et entre le diplôme et le salaire se sont distendues pour les jeunes générations dotées d'une ancienneté inférieure à dix ans (Nauze-Fichet et Tomasini, 2002, p. 22). Au niveau macroéconomique, le déclassement apparait, parallèlement au chômage, comme une forme de sous-emploi liée à une pénurie d'emplois qualifiés (Giret, NauzeFichet et Tomasini, 2006, p. 306). Utilisant la méthode statistique pour mesurer le déclassement, les auteures mettent en évidence un déplacement des diplômes durant les années 1990 vers des emplois de moins en moins qualifiés (Nauze-Fichet et Tomasini, 2002, p. 33). Elles poursuivent leur recherche en développant une approche du déclassement par les salaires. Cette stratégie permet de contourner les problèmes rencontrés pour mesurer la relation entre la formation et l'emploi d'un même individu et elle met en évidence les déterminants du déclassement. Ceux-ci sont de trois ordres: des facteurs individuels sociodémographiques, des facteurs individuels décrivant l'insertion sur le marché du travail (type de contrat, etc.) et des facteurs environnementaux. Tout d'abord, les caractéristiques sociodémographiques ont une incidence sur le risque de déclassement salarial: être une femme ou vivre seul accroissent le risque. La vie en couple a également des effets différents selon le statut du conjoint (8). Être issu d'une famille dont le père est cadre préserve du déclassement salarial, grâce à une meilleure connaissance du fonctionnement des négociations salariales, mais aussi grâce au capital social. Ensuite, le passé de l'individu sur le marché de l'emploi joue un rôle déterminant: moins on est inséré, plus le risque de déclassement est élevé; à l'inverse, la menace est moindre lorsqu'on était en contrat à durée indéterminée auparavant. Enfin, l'environnement professionnel du travailleur n'est pas à négliger : le commerce et 
l'agriculture sont, en France, les secteurs où le risque de déclassement est le plus important.

Dans une autre publication, Nauze-Fichet et Tomasini (2005) poursuivent l'étude du déclassement salarial en s'intéressant au parcours des individus durant les trois années qui suivent la sortie du système de formation. De plus en plus de jeunes acceptent le déclassement afin d'obtenir un emploi, ainsi qu'une baisse de salaire, pour passer d'un emploi précaire à un emploi stable. Le reclassement se fait dans six cas sur dix par une mobilité externe et non par une promotion interne. Identifiant de nouveau les facteurs qui concourent au reclassement, les auteurs montrent que celui-ci est toujours lié aux origines sociales de l'individu et au rôle des réseaux sociaux sur le marché du travail (Nauze-Fichet et Tomasini, 2005, p. 68).

Dirigé par Giret, Lopez et Rose (2005), l'ouvrage intitulé Des formations pour quels emplois? présente les résultats de l'enquête Génération 98 du Centre d'études de recherches sur l'emploi et les qualifications (CÉREQ), qui porte sur les caractéristiques des emplois occupés au cours des premières années de vie active. L'allongement des durées d'études semble s'essouffler. Entre 1995 et 2001, les effectifs de l'enseignement supérieur n'ont pas progressé et, s'ils ont légèrement augmenté depuis, cela provient en partie de l'arrivée d'étudiants étrangers. Couppié, Giret et Lopez (2005) observent que la correspondance entre les formations initiales et les premiers emplois est mal assurée. Plus d'un jeune sur deux trouve un emploi nécessitant moins de qualifications que ne lui en donne sa formation. Pour le total des jeunes diplômés, le taux de déclassement atteint 32\% pour les sortants de 1980, $33 \%$ pour ceux de 1990, $46 \%$ pour ceux de 1995 et $43 \%$ chez les sortants de 1998. Ce sont les femmes qui sont les plus concernées par cette situation: en 1998, un homme sur trois et plus d'une femme sur deux sont déclassés, parmi les sortants de 1998. Enfin, le phénomène concerne avant tout les diplômés de niveau bac et bac +2 .

Bien souvent, le premier emploi est sans rapport avec le type de formation suivie (Couppié, Giret et Lopez, 2005, p. 79). Cumulés au déclassement, ces désajustements en termes de spécialité concernent $60 \%$ des premiers emplois.

L'approche subjective est également utilisée. La part des jeunes se déclarant employés au-dessous de leur niveau de compétences est importante chez les bac + 1 ou 2 non diplômés, c'est-à-dire après 13 ou 14 années d'études (39\%); les diplômées du troisième cycle de sciences humaines et sociales, après 21 années d'études (34\%), puis chez les bacheliers non diplômés et les bacheliers professionnels, techniques et généraux, qui comptent 12 années d'études (32\%) (Giret, 2005, p. 283). En conclusion, l'approche subjective nous permet d'apprendre que près d'un jeune sur trois estime être employé en-dessous de son niveau de compétences.

Dans le même ouvrage, Béduwé (2005) montre que le niveau de formation initiale et de diplôme des salariés recrutés sur des emplois désignés comme non qualifiés ne cesse de progresser. Dans un contexte de chômage massif et en raison 
de l'élévation du niveau d'embauche, de plus en plus de jeunes diplômés se trouvent relégués au salariat d'exécution. Le départ de l'emploi non qualifié pour ces jeunes n'est pas si fréquent. Six trajectoires sont repérées: si pour certains, les plus qualifiés, ce type d'emploi ne constitue qu'une étape transitoire, les plus faiblement diplômés ont moins de chances d'évolution, et nombreux sont ceux qui s'établissent dans l'emploi ou accumulent des séjours brefs dans plusieurs emplois non qualifiés (Béduwé, 2005, p. 358).

Bien que nous ayons restreint notre périmètre d'investigation, nous devons ajouter que le phénomène de déclassement dans l'accès à l'emploi est une situation de plus en plus fréquente au-delà de la France et de l'Amérique du Nord. Pour ce qui est des pays européens, Brynin (2002, p. 238) observe par exemple une croissance de la sur-éducation dans l'emploi au Portugal, spécialement entre 1985 et 1992, et aux Pays-Bas entre 1960 et 1995. En Grande-Bretagne, 36\% des hommes et $41 \%$ des femmes sont surdiplômés dans leur premier emploi (Dolton et Vignoles, 2000, p.184).

\section{Conclusion}

En plus de donner des informations sur la croissance du déclassement dans l'accès à l'emploi et les variations du phénomène selon le sexe de la personne, le niveau d'études atteint et la spécialité de formation, l'ensemble de ces publications permet de saisir et d'illustrer une partie des transformations de l'espace social, comme l'apparition massive de la figure du surdiplômé. Ce qu'il convient de retenir, c'est que l'insertion professionnelle pour les jeunes populations ne dépend pas seulement des choix et faits de l'individu. La pénurie globale d'emplois (qualifiés) liée à l'insuffisance de la demande joue un rôle primordial. D'ailleurs, le mouvement de déclassement est plus net encore depuis le début des années 1990, c'est-à-dire à mesure que s'accroît et perdure le chômage. Il est, en outre, renforcé par le fait que les reclassements en cours de carrière ainsi que l'accès à un contrat à durée indéterminée sont désormais moins fréquents ou de plus en plus lents, compte tenu de la précarisation du travail, en France comme en Amérique du Nord (Gauthier, 1996, p. 142-144; Givord, 2005).

Le processus de déclassement est surtout important pour ce qu'il génère. Que modifie l'installation de générations diplômées dans des emplois qui ne correspondent pas à la valeur de leur titre scolaire (déclassement) ou encore à leur spécialité de qualification (inadaptation) ? De quelle façon ces strates de la population vont-elles s'établir dans l'emploi? Du côté des employeurs, l'enjeu est paradoxal. D’une part, le recrutement de salariés diplômés pour des emplois ne nécessitant pas autant de qualifications que celles détenues par les individus peut paraître intéressant à court terme du point de vue de la productivité, si l'on retient les analyses de Vahey (2000) sur le maintien d'une productivité au travail des surqualifiés et non celles de Berg (1970) qui démontraient le contraire. Comme l'ont montré Baudelot et Establet (2000, p. 162-163) pour l'emploi dans les fast- 
food, les employeurs tirent un bénéfice des attributs sociaux des anciens étudiants tels que la polyvalence, une bonne présentation de soi, la diction socialement normée et l'intériorisation de l'ordre social. D'autre part, nous pouvons nous interroger à long terme sur l'enracinement au travail et l'engagement professionnel de ces jeunes déclassés qui, après un passage prolongé dans l'enseignement supérieur, espèrent obtenir un emploi à la hauteur de leur qualification. Une telle forme d'engagement professionnel est-elle économiquement et socialement rentable à long terme pour les entreprises qui, profitant de l'afflux massif de diplômés et des difficultés d'accès à l'emploi, recrutent des jeunes à des postes peu qualifiés? Dans ces types d'emplois au sein desquels la part de diplômés augmente, quels motifs de satisfaction au travail et quelles zones d'autonomie les surqualifiés peuvent-ils trouver afin de gérer au mieux l'expérience du déclassement? Nul doute que des enquêtes réalisées dans plusieurs secteurs d'activité apporteront quelques réponses à ces questions (Chabault, 2007).

\section{Notes}

1. Déclassement et sur-éducation désignent ici le même phénomène. Dans les publications canadiennes (anglophones et francophones) et états-uniennes, les termes overeducation (ou overschooling) ou sur-éducation sont fréquemment utilisés.

2. The mismatch between educational level and the educational requirements of the occupation (Smith, 1986, 90).

3. Dumartin précise: cette appréhension des rapports entre système éducatif et système productif est relativement nouvelle: jusqu'aux années 1970 en France, dominait la conviction que les prévisions macroéconomiques pouvaient indiquer les besoins futurs en qualification. Elle ajoute que les travaux français des V $V^{e}$ et VI ${ }^{e}$ Plans couvrant la période 1966-1975 étaient marqués par la tentation de mettre en adéquation les flux de formation avec les besoins de la Nation. Cette approche comporte des lacunes: d'une part, les individus ne se comportent pas selon les orientations conseillées par ces rapports et, d'autre part, le déroulement des carrières amène souvent des actifs loin de leur domaine de formation initiale (Dumartin, 1997, 60).

4. Le Centre d'études et de recherches sur l'emploi et les qualifications réalisent, depuis 1992, en France, des enquêtes générationnelles dont le principal objectif est de saisir les cheminements professionnels en début de vie active d'une cohorte d'individus questionnés après la fin de leurs études. L'enquête Génération 98 s'intéresse aux 750000 jeunes sortis de formation initiale en 1998 et 56000 jeunes ont été une première fois interrogés en 2001, puis de nouveau en 2003.

5. Voir Tanguy, 1986; Gautié et Nauze-Fichet, 2000.

6. Les conclusions de Vultur sont alors semblables à celles de Gautié et Nauze-Fichet pour la France $(2000,287)$.

7. Ou signalement, pour reprendre le terme introduit par Arrow (1973). Selon cet auteur, le diplôme signale aux employeurs les qualités des travailleurs, même si elles doivent peu à l'instruction reçue. 
SUMmARY - Since the 1980s, overeducation has characterized access and integration into the job market for young adults both in France and in North America. This article aims to study French and North American publications dealing with this sociological problem. First, the author shows the different approaches for measuring the phenomenon, and then presents the results of statistical surveys from France and North America dating from the 1970s to 2000 s.

KEY WORDS - job market integration, young generations, degrees, overeducation, France, North America.

RESUMEN • El fenómeno de la desclasificación (o supra-educación) en cuanto al acceso al empleo caracteriza la inserción profesional de las nuevas poblaciones, en Francia como en Norteamérica, desde hace dos décadas. En esta perspectiva, el presente artículo presenta una evaluación de la situación de las publicaciones norteamericanas y francesas acerca de este fenómeno. Una vez presentados los diferentes enfoques destinados a medir este fenómeno, exponemos los resultados de unas encuestas estadísticas canadienses, americanas y francesas desde los años 1970 hasta los años 2000.

PALABRAS CLAVES • inserción profesional, nuevas generaciones, diplomas, reclasificación, Francia, Norteamérica.

\section{Références}

Affichard, J. (1981). Quels emplois après l'école? La valeur des titres scolaires depuis 1973. Économie et statistique, 134, 7-26.

Arrow, K. J. (1973). Higher Education as a Filter. Journal of Publics Economics, 2(3), 193216.

Baudelot, C. et Establet, R. (2000). Avoir trente ans en 1968 et 1998. Paris, France: Seuil.

Beaud, S. (2000). Jeunes ouvriers bacheliers. Sur le déclassement des enfants de la démocratisation. Lien social et politiques - RIAC, 43, 103-112.

Béduwé, C. (2005). Peut-on parler de relation formation-emploi au sein des emplois non qualifiés? Dans J.-F. Giret, A. Lopez et J. Rose (Dir.) : Des formations pour quels emplois? Paris, France: La Découverte/Céreq.

Berg, I. (1970). Education and jobs: the great training robbery. New York, New York: Praeger.

Bourdieu, P. (1978). Classement, déclassement, reclassement. Actes de la recherche en sciences sociales, $18,2-22$.

Brynin, M. (2002). Overqualification in employment. Work, Employment and Society, 16(4), 637-654.

Burris, V. (1983). The social and political consequences of overeducation. American Sociological Review, 48(4), 454-467.

Cartier, M. (2003). Les facteurs et leurs tournées. Un service public au quotidien. Paris, France: La Découverte.

Chabault, V. (2007). Entre le commerce et la culture. Les pratiques de travail des vendeurs de livres de la FNAC. Sociétés contemporaines, 66, 27-46. 
Chauvel, L. (1998a). La seconde explosion scolaire: diffusion des diplômes, structure sociale et valeur des titres. Revue de l'OFCE, 66, 5-36.

Chauvel, L. (1998b). Le destin des générations. Structure sociale et cohortes en France au $\mathrm{XX}^{\mathrm{e}}$ siècle. Paris, France: Presses universitaires de France.

Couppié, T., Giret, J.-F. et Lopez, A. (2005). Des formations initiales aux premiers emplois : une correspondance plutôt mal assurée. Dans J.-F. Giret, A. Lopez et J. Rose (Dir.): Des formations pour quels emplois? Paris, France: La Découverte.

Dolton, P., and Vignoles, A. (2000). The incidence and effects of overeducation in the UK Graduate Labour Market. Economics of Education Review, 19(2), 179-198.

Dumartin, S. (1997). Formation-emploi: quelle adéquation? Économie et statistique, 303, 59-80.

Duru-Bellat, M. (2006). L'inflation scolaire. Les désillusions de la méritocratie. Paris, France: Seuil.

Forgeot, G. et Gautié, J. (1996). Chômage des jeunes et relation formation emploi. La lettre du CEE, 43, 1-9.

Forgeot, G. et Gautié, J. (1997). Insertion professionnelle des jeunes et processus de déclassement. Économie et statistique, 304-305, 53-74.

Freeman, R. (1976). The overeducated American. New York, New York: Academic Press.

Frenette, M. (2000). Les employés surqualifiés? Les diplômés récents et les besoins de leurs employeurs. Revue trimestrielle de l'éducation, 7(1), 7-22.

Gauthier, M. (1996). Précaires un jour... ? Ou quelques questions à propos de l'avenir des jeunes contemporains. Sociologie et sociétés, 28(1), 135-146.

Gautié, J. et Nauze-Fichet, E. (2000). Déclassement sur le marché du travail et retour au plein emploi. Rapport Pisani-Ferry, Conseil d'analyse économique. Paris, France: La Documentation française.

Giret, J.-F. (2005). Quand les jeunes s'estiment déclassés. Dans J.-F. Giret, A. Lopez et J. Rose (Dir.): Des formations pour quels emplois? Paris, France: La Découverte.

Giret, J.-F., Lopez, A. et Rose, J. (2005). Des formations pour quels emplois? Paris, France: La Découverte.

Giret, J.-F., Nauze-Fichet, E. et Tomasini, M. (2006). Le déclassement des jeunes sur le marché du travail. Données sociales. La société française, INSÉÉ, 306-314.

Givord, P. (2005). Formes particulières d'emploi et insertion des jeunes. Économie et statistique, 388-389, 129-144.

Guérin, G., Carrière, J. et Wils, T. (1999). Facteurs explicatifs de la démobilisation chez les diplômés universitaires récemment embauchés. Relations industrielles/Industrial relations, 54(4), 643-672.

Langlois, S. (2003). Quatre décennies d'études sur la stratification sociale au Québec et au Canada: tendances et illustrations. Lien social et Politiques - RIAC, 49, 45-70.

Lefresne, F. (2003). Les jeunes et l'emploi. Paris, France: La Découverte.

Montmarquette, C. et Thomas, L. (2003). Surqualification et sous-qualification des travailleurs sur le marché du travail: le cas du Québec et de l'Ontario en 1991 et 1996. Montréal, Québec: Centre interuniversitaire de recherche en analyse des organisations. 
Nauze-Fichet, E. et Tomasini, M. (2002). Diplôme et insertion sur le marché du travail: approches socioprofessionnelle et salariale du déclassement. Économie et statistique, 354, 21-43.

Nauze-Fichet, E. et Tomasini, M. (2005). Parcours des jeunes à la sortie du système éducatif et déclassement salarial. Économie et statistique, 388-389, 57-84.

Passeron, J.-C. (1982). L'inflation des diplômes. Remarques sur l'usage de quelques concepts analogiques en sociologie. Revue française de sociologie, 23(4), 551-584.

Rose, J. (1998). Les Jeunes face à l'emploi. Paris, France: Desclée de Brouwer.

Saint-Pierre, C. (2001). L'insertion professionnelle et citoyenne des jeunes au Québec. Dans M. Gauthier et L. Roulleau-Berger (Dir.): Les jeunes et l'emploi dans les villes d'Europe et d'Amérique du Nord. La Tour d'Aigues, France: Éditions de l'Aube.

Schwartz, O. (1998). La notion de «classes populaires». Habilitation à diriger des recherches en sociologie. Saint Quentin en Yvelines, France: Université de Versailles.

Sicherman, N. (1991). Overeducation in the Labor Market. Journal of Labor Economics, $9(2), 101-122$.

Smith, H. L. (1986). Overeducation and underemployement: an agnostic review. Sociology of Education, 59(2), 85-99.

Tanguy, L. (1986). L'introuvable relation formation/emploi, état d'un champ de recherche en France. Paris, France: La Documentation française.

Thurow, L. (1975). Generating Inequality. New York, New York: Basic Books.

Vahey, S. P. (2000). The great Canadian training robbery: evidence on the returns to educational mismatch. Economics of Education Review, 19(2), 219-227.

Vultur, M. (2003). L'insertion sociale et professionnelle des jeunes au Québec. Évolution et situation actuelle. Dans M. Gauthier (Dir.) : Regard sur... La jeunesse au Québec. SainteFoy, Québec: éditions de l'IQRC/PUL.

Vultur, M. (2006). Diplôme et marché du travail. La dynamique de l'éducation et le déclassement au Québec. Recherches sociographiques, 47(1), 41-68.

Texte reçu le: 6 septembre 2006

Version finale reçue le: 9 juillet 2007

Accepté le: 22 novembre 2007 